\title{
Procyanidin B2 and a cocoa polyphenolic extract inhibit acrylamide- induced apoptosis in human Caco-2 cells by preventing oxidative stress and activation of JNK pathway. \\ Ildefonso Rodríguez-Ramiro, Sonia Ramos, Laura Bravo, Luis Goya, María Ángeles Martín
}

Department of Metabolism and Nutrition, Instituto del Frío-ICTAN, Consejo Superior de Investigaciones Científicas (CSIC), Madrid, Spain

* Corresponding author:

Department of Metabolism and Nutrition, Instituto del Frío-ICTAN, Consejo Superior de Investigaciones Científicas, José Antonio Novais 10, Ciudad Universitaria, 28040, Madrid, Spain. Phone: +34.91.544.56.07. Fax:+34.91.549.36.27 e-mail: amartina@,if.csic.es

Running tittle: Cocoa polyphenols inhibit AA toxicity in Caco-2 cells

This work was supported by the grant AGL2007-64042/ALI and project CSD 200700063 from Programa Consolider-Ingenio from the Spanish Ministry of Science and Innovation (CICYT). I. Rodriguez-Ramiro is a predoctoral fellow of the Consejo Superior de Investigaciones Científicas.

Keywords: Glutathione, epicatechin, ROS, signalling pathways. 


\section{Abstract}

Humans are exposed to dietary acrylamide (AA) during their lifetime, it is therefore necessary to investigate the mechanisms associated with AA induced toxic effects. Accumulating evidence indicates that oxidative stress may contribute to AA cytotoxicity but the link between oxidative stress and AA cytotoxicity in the gastrointestinal tract, the primary organ in contact with dietary AA, has not been described. In this study, we evaluate the alterations of the redox balance induced by AA in Caco-2 intestinal cells as well as the potential protective role of natural antioxidants such as a well-standardized cocoa polyphenolic extract (CPE) and its main polyphenol components epicatechin (EC) and procyanidin $\mathrm{B} 2$ (PB2). We found that AA-induced oxidative stress in Caco-2 cells is evidenced by glutathione (GSH) depletion and reactive oxygen species (ROS) overproduction. AA also activated the extracellular-regulated kinases (ERKs) and the c-jun $\mathrm{N}$-amino terminal kinases (JNKs) leading to an increase in caspase-3 activity and cell death. Studies with appropriate inhibitors confirmed the implication of oxidative stress and JNKs activation in AA-induced apoptosis. Additionally, AA cytotoxicity was counteracted by CPE or PB2 by inhibiting GSH consumption and ROS generation, increasing the levels

of gamma-glutamyl cysteine synthase ( $\gamma$-GCS) and glutathione-S-transferase (GST) and blocking the apoptotic pathways activated by AA. Therefore, AA-induced cytotoxicity and apoptosis are closely related to oxidative stress in Caco-2 cells. Interestingly, natural dietary antioxidant such as PB2 and CPE were able to suppress AA toxicity by improving the redox status of Caco- 2 cells and by blocking the apoptotic pathway activated by AA. 


\section{Introduction}

In the last years, relevant amounts of acrylamide (AA) have been identified in heattreated carbohydrate-rich foods such as fried potatoes, cookies, bread and breakfast cereals [1]. Extensive in vivo and in vitro studies have revealed that AA could induce neurotoxic, genotoxic, carcinogenic, developmental, and reproductive toxic effects [2]. Therefore, the presence of AA in so commonly ingested foods has originated a great concern because humans could be exposed to significant quantities of AA during their life-span.

Most investigations of AA toxicity have mainly focused on their genotoxic and carcinogenic properties; however, accumulating evidences [3-6] seem to indicate that AA also possesses cytotoxic properties by affecting the redox status of the cells. Exposure to AA induces a significant reduction in GSH concentration in different cells [7-9] and contributed to the onset of oxidative stress conditions, which can adversely affect cell function and potentially lead to cell damage and death [10]. However, while previous studies have documented the link between AA-induced oxidative stress and cytotoxicity in several tissues [3-5], to date the potential impact of AA on the integrity of gastrointestinal tract, the primary organ in contact with food, is poorly understood.

AA is readily absorbed into the intestinal cells and it is usually non-enzymatically and enzymatically conjugated with glutathione (GSH) resulting in a depletion of cellular GSH stores [11]. The decreased GSH levels, as occurs under various pathological states [12], increase the levels of reactive oxygen species (ROS) which are widely recognized as important mediators of cell function [10]. Hence, increased ROS have been shown to activate signalling cascades involving the MAPK-JNKs that play a key role in the regulation of many cellular processes, including apoptosis [10]. Interestingly, studies in intestinal cells $[13,14]$ have demonstrated that loss of cellular glutathione redox balance is 
an important player in apoptotic signalling and cell death. Therefore, depletion of GSH levels favouring cellular oxidative stress and apoptosis may be suggested as a potential mechanism for AA toxicity.

Considering this, the use of natural compounds derived from the diet with antioxidant effects might provide a strategy to reduce AA toxicity. Among dietary compounds, cocoa and its polyphenol components epicatechin, catechin and procyanidins have exhibited beneficial effects on numerous oxidative-stress related diseases [15]. In addition, cocoa derived products are commonly consumed in many countries in the European Union and the United States [16]. Thus, they can be considered as dietary antioxidants and as natural products with beneficial properties. Cocoa polyphenols have shown to exert their protective action as reducing agents by improving antioxidant defences through the induction of antioxidant and phase II enzymes [17] and also through its ability to modulate certain signalling pathways involved in cell survival and death [18-20]. Furthermore, the fact that the intestinal epithelium can be exposed to high concentrations of some of these polyphenols implies that they could have an important role in the prevention of the potential oxidative stress induced by AA.

Therefore, the aim of the present study was to investigate whether AA is able to induce oxidative stress and apoptosis in Caco- 2 cells, a human cell line originating from the gastrointestinal tract that retains many of the morphological and enzymatic features typical of normal human enterocytes. In addition, the likely protective effects on this process of a cocoa polyphenolic extract (CPE) and its polyphenol components epicatechin (EC) and procyanidin $\mathrm{B} 2$ (PB2) individually were also evaluated. 


\section{Material and Methods}

\subsection{Materials and Chemical}

EC, N-acetylcysteine (NAC), o-phthalaldehyde (OPT), gentamicin, penicillin G, streptomycin, acrylamide, PD98059 [2-(2-amino-3-methoxyphenyl)-4H-1-benzopyran-4one] and SP600125 [Anthra-(1,9-cd)pyrazol-6(2H)-one] were purchased from Sigma Chemicals (Madrid, Spain). The fluorescent probe 2',7'-dichlorofluorescin diacetate (DCFH-DA) was from Molecular Probes (Eugene, OR). PB2 was purchased from Extrasynthese (Genay, France). Anti-ERKs, antiphospho-ERKs (p-ERKs), anti-JNKs, antiphospho-JNK (p-JNKs) and anti-ß-actin were obtained from Cell Signaling Technology (Izasa, Madrid, Spain). Anti- $\gamma$-GCS and anti-GST were purchased from Santa Cruz (Quimigen, Madrid, Spain). Caspase-3 substrate (Ac-DEVD-AMC) was purchased from Pharmingen (San Diego, CA). Materials and chemicals for electrophoresis and the Bradford reagent were from BioRad (Madrid, Spain). Cell culture dishes were from Falcon (Cajal, Madrid, Spain) and cell culture medium and fetal bovine serum (FBS) from Biowhittaker Europe (Innogenetics, Madrid, Spain).

\subsection{Cocoa polyphenol extraction}

Natural Forastero cocoa powder (Nutrexpa, Barcelona, Spain) was used for this study. Soluble polyphenols were extracted by sequentially washing $1 \mathrm{~g}$ of sample with 40 $\mathrm{mL}$ of $16 \mathrm{mM}$ hydrochloric acid in $50 \%$ aqueous methanol (50:50, v/v, 1 hour at room temperature, constant shaking) and $40 \mathrm{~mL}$ of acetone-water $(70: 30, \mathrm{v} / \mathrm{v}, 1$ hour room temperature, constant shaking) in $50 \mathrm{~mL}$ centrifuge tubes [17]. After centrifugation (15min, $3000 \mathrm{~g}$ ), supernatants from each extraction step were combined and made up to $100 \mathrm{~mL}$. 
The desiccated extract was dissolved in distilled water and kept frozen until assay. The total polyphenol content was determined by the spectrophotometric method of Folin-Ciocalteau [17] using gallic acid as standard. A Beckman DU640 spectrophotometer (Beckman Instruments Inc., Fullerton, CA) was used. Analysis of the CPE by LC-MS showed that the extract is composed mostly of epicatechin $(383.5 \mathrm{mg} / 100 \mathrm{~g})$, catechin $(116 \mathrm{mg} / 100 \mathrm{~g})$ and procyanidins $(254.5 \mathrm{mg} / 100 \mathrm{~g})$ and non-flavonoid compounds such as theobromine [17].

\subsection{Cell culture and AA and polyphenols treatments}

Human Caco-2 cells were grown in a humidified incubator containing $5 \% \mathrm{CO}_{2}$ and $95 \%$ air at $37{ }^{\circ} \mathrm{C}$. They were grown in DMEM F-12 medium from Biowhitaker (Lonza, Madrid, Spain), supplemented with $10 \%$ Biowhitaker foetal bovine serum (FBS) and 50 $\mathrm{mg} / \mathrm{L}$ of each of the following antibiotics: gentamicin, penicillin and streptomycin. Plates were changed to FBS-free medium the day before the assay

For the AA treatment, different concentrations of AA $(1,5,10$ and $20 \mathrm{mM})$, diluted in serum-free culture medium and filtered through a $0.2 \mu \mathrm{m}$ membrane, were added to the cell plates for different times. In the experiments with the pharmacological inhibitors, cells were preincubated with $1 \mathrm{mM}$ of NAC (a GSH precursor) or with $50 \mu \mathrm{M}$ PD98059 (a specific inhibitor of MAPK-ERK kinase) or with $50 \mu \mathrm{M}$ of SP600125 (a specific inhibitor of JNKs) for $1 \mathrm{~h}$ before and during AA treatment.

To evaluate the protective effect of cocoa and cocoa flavonoids against AA toxicity, $10 \mu \mathrm{M}$ of EC or PB2 or $10 \mu \mathrm{g} / \mathrm{mL}$ of CPE diluted in serum-free culture medium and filtered through a $0.2 \mu \mathrm{m}$ membrane, were added to the cell plates for 20 hours. After that, the medium was discarded and fresh medium containing $5 \mathrm{mM}$ of AA was added for different times. 


\subsection{Cytotoxicity assays}

Cellular damage induced by AA was evaluated by crystal violet assay [20] and by lactate dehydrogenase (LDH) leakage [21]. For the crystal violet assay, Caco-2 cells were seeded at low density $\left(10^{4}\right.$ cells per well) in 96-well plates, grown for 20 hours with the different treatments and incubated with crystal violet $(0.2 \%$ in ethanol) for $20 \mathrm{~min}$. Plates were rinsed with distilled water, allowed to dry, and 1\% sodium dodecyl sulfate (SDS) added. The absorbance of each well was measured using a microplate reader at $570 \mathrm{~nm}$ (Bio-Tek, Winooski, VT, USA). For LDH assay, the culture medium and the cells scraped in PBS were collected after the different treatments. Cells were first sonicated to ensure breaking down the cell membrane to release the total amount of LDH; then, after centrifugation $(1000 \mathrm{~g}, 15 \mathrm{~min})$ to clear up the cell sample, $10 \mu \mathrm{L}$ were placed into a well of a 96 multiwell for the assay. In the same manner, $10 \mu \mathrm{L}$ of each culture medium were also deposited into a well of a 96-well multiwell. The LDH leakage percentage was estimated from the ratio between the LDH activity in the culture medium and that of the whole cell content.

\subsection{Apoptosis}

Apoptosis was evaluated as activation of Caspase-3 [22]. Cells were lysed in a buffer containing $5 \mathrm{mM}$ Tris (pH 8), $20 \mathrm{mM}$ EDTA, and $0.5 \%$ Triton X-100. The reaction mixture contained $20 \mathrm{mM}$ HEPES (pH 7), $10 \%$ glycerol, $2 \mathrm{mM}$ dithiothreitol (DTT), and $30 \mu \mathrm{g}$ of protein per condition, and $20 \mu \mathrm{M}$ Ac-DEVDAMC ( $N$-acetyl-Asp-Glu-Val-Asp-7amino-4-methylcoumarin) as substrate. Enzymatic activity was determined by measuring fluorescence at an excitation wavelength of $380 \mathrm{~nm}$ and an emission wavelength of $440 \mathrm{~nm}$ (Bio-Tek, Winooski, VT, USA). 


\subsection{ROS generation}

Cellular ROS were quantified by the DCFH assay using a microplate reader. For the assay, cells were plated in 24 -well multiwells at a rate of $2 \times 10^{5}$ cells per well and changed to FBS-free medium and the different treatments the day before the assay. After that, $5 \mu \mathrm{M}$ DCFH was added to the wells for $30 \mathrm{~min}$ at $37^{\circ} \mathrm{C}$. Then, cells were washed twice with PBS and $0.5 \mathrm{~mL}$ of serum-free medium or serum-free medium with AA were added per well. After being oxidized by intracellular oxidants, DCFH will become dichorofluorescein (DCF) and emit fluorescence. ROS generation was evaluated at different times in a fluorescent microplate reader at an excitation wavelength of $485 \mathrm{~nm}$ and an emission wavelength of $530 \mathrm{~nm}$ (Bio-Tek, Winooski, VT, USA).

\subsection{Determination of Reduced Glutathione (GSH)}

The content of GSH was quantified by the fluorometric assay of Hissin and Hilf [23]. The method takes advantage of the reaction of GSH with OPT at $\mathrm{pH}$ 8.0. After the different treatments, the culture medium was removed and cells were detached and homogenized by ultrasound with $5 \%$ trichloroacetic acid containing $2 \mathrm{mM}$ EDTA. Following centrifugation of cells for $30 \mathrm{~min}$ at $3000 \mathrm{rpm}, 50 \mu \mathrm{L}$ of the clear supernatant were transferred to a 96 multiwell plate for the assay. Fluorescence was measured at an excitation wavelength of $340 \mathrm{~nm}$ and an emission wavelength of $460 \mathrm{~nm}$. The results of the samples were referred to those of a standard curve of GSH. The precise protocol has been described elsewhere [21].

\subsection{Preparation of cell lysates for Western blotting}


To detect the levels of $\gamma$-GCS, GST, ERKs, p-ERKs, JNKs and p-JNKs, cells were lysed at $4{ }^{\circ} \mathrm{C}$ in a buffer containing $25 \mathrm{mM}$ HEPES (pH 7.5), $0.3 \mathrm{M} \mathrm{NaCl}, 1.5 \mathrm{mM} \mathrm{MgCl}$, 0.2 mM EDTA, 0.5 mM DTT, 0.1\% Triton X-100, $200 \mathrm{mM}$ ß-glycerolphosphate, $0.1 \mathrm{mM}$ $\mathrm{Na}_{3} \mathrm{VO}_{4}, 21 \mathrm{~g} / \mathrm{mL}$ leupeptin, and $1 \mathrm{mM}$ phenylmethylsulphonyl fluoride (PMSF). The supernatants were collected, assayed for protein concentration by using the Bradford reagents, aliquoted and stored at $-80{ }^{\circ} \mathrm{C}$ until used for Western blot analyses.

\subsection{Protein determination by Western Blotting}

Equal amounts of protein $(100 \mu \mathrm{g})$ were separated by SDS-PAGE and transferred to polyvinylidene difluoride (PVDF) filters (Protein Sequencing Membrane, Millipore). Membranes were probed with the corresponding primary antibody followed by incubation with peroxide-conjugated antirabbit Ig (GE Healthcare, Madrid, Spain) or peroxideconjugated antimouse IgG (Sigma, Madrid, Spain) for GST antibody. Blots were developed with the ECL system (GE Healthcare). Normalization of Western blot was ensured by ßactin and bands were quantified by laser scanning densitometry (Molecular Dynamics, Sunnyvale, CA).

\subsection{Statistics}

Statistical analysis of data was as follows: prior to analysis the data were tested for homogeneity of variances by the test of Levene; for multiple comparisons, one-way ANOVA was followed by a Bonferroni test when variances were homogeneous or by the

Tamhane test when variances were not homogeneous. The level of significance was $\mathrm{P}<$ 0.05. A SPSS version 17.0 program has been used. 


\section{3.- Results}

\subsection{Effect of AA on cell viability and apoptosis in Caco2 cells.}

In order to identify the doses at which cell toxicity was induced by AA, Caco-2 cells were exposed during $16 \mathrm{~h}$ at different concentrations of AA (1-20 mM). As shown in Fig. 1A, cell viability, as determined by the crystal violet assay, was significantly decreased in the presence of 5-20 mM AA. Comparable results were obtained when cytotoxicity was evaluated by the LDH leakage; AA induced a dose-dependent increase in toxicity (Fig. 1B). Additionally, time course experiments reveals that lower concentrations of AA $(<5 \mathrm{mM})$ decreased cell viability of Caco- 2 cells but for a longer exposure times ( $>36$ hours) (data not shown).

To further determine whether the cytotoxic effect was to some extent due to apoptosis, Caco-2 cells were exposed to $1-20 \mathrm{mM}$ of AA for $8 \mathrm{~h}$ and caspase- 3 activity was analyzed as a biomarker of apoptosis. As shown in Figure 1C, the presence of 5-20 mM AA induced the activation of caspase-3, which reached an activation peak at the dose of 5 $\mathrm{mM}$ at this time. Similarly to what was found in cell viability, time-course experiments showed that doses of 10 and $20 \mathrm{mM}$ AA achieved the highest caspase- 3 activation at shorter exposure time $(<8 \mathrm{~h})$ while $1 \mathrm{mM}$ AA significantly increased caspase- 3 activity but for exposures longer than $24 \mathrm{~h}$ (data not shown). Altogether, these results indicate that AA is able to activate caspase- 3 and thus to induce apoptosis and cell death in Caco-2 cells.

\subsection{AA decreases GSH levels and induces ROS generation in Caco2 cells.}

Since cell death by apoptosis can be considered the final event of a redox imbalance, we next elucidated the redox status of Caco-2 cells evaluating GSH levels and ROS generation after AA treatment (0-4 h). Figure 2A showed that GSH content rapidly 
decreased during incubation with 1-20 mM AA. Significant decreases were observed as early as $1 \mathrm{~h}$, and GSH levels in the presence of 5-20 mM AA were less than 50\% and $20 \%$ of control after 2 and $4 \mathrm{~h}$, respectively. The decrease in GSH levels occurred in AA concentration- and time-dependent manner. Accordingly, 5-20 mM AA caused a significant increase in ROS production after $2 \mathrm{~h}$ of exposure (Fig. 2B), showing a two fold increase at $5 \mathrm{mM}$ and a three fold at $20 \mathrm{mM}$. Conversely, ROS generation in the presence of $1 \mathrm{mM} \mathrm{AA}$ was significantly increased only after 4 hours of treatment. These results show that AA induces a redox imbalance in Caco-2 cells by decreasing GSH levels and increasing ROS generation.

Given that $5 \mathrm{mM}$ of AA was the smallest concentration that provoked cytotoxicity at the shortest time and induced GSH depletion, ROS generation and activation of caspase3 , it was the concentration selected for the following experiments.

\subsection{AA activates ERKs and JNKs in Caco-2 cells.}

Since oxidative stress is known to activate members of the MAPK family involved in the regulation of apoptosis, we next investigated the effect of AA on the expression and activities of two important constituents of this signalling cascade, the ERKs and JNKs kinases. Total and phosphorylated (active form) levels of ERKs and JNKs were evaluated by Western blotting at different times (0-16 h) of AA exposure. As shown in Fig. 3, the levels of p-ERKs increased at 2 hours of AA-treatment reaching a peak at $4 \mathrm{~h}$ before decreasing at a longer incubation time. Conversely, phosphorylation of JNKs was only

evident after $4 \mathrm{~h}$ of treatment and remained active until $16 \mathrm{~h}$. Total ERKs and JNKs protein levels did not change during AA exposure. These results indicate that AA-induced 
apoptosis and cytotoxicity in Caco-2 cells is accompanied by an early and transient increase of p-ERKs and a sustained increase of p-JNKs.

\subsection{Oxidative stress and JNKs activation are implicated on apoptosis and cytotoxicity} induced by $A A$.

Next, we aimed to elucidate whether AA-induced apoptosis and cell cytotoxicity could be associated with the altered cellular redox status and/or ERKs and JNKs activation observed in AA-treated cells. To this end, we examined the effect of the strong antioxidant NAC (a GSH synthesis precursor and ROS scavenger) and of the pharmacological ERKs and JNKs inhibitors, PD98059 (PD) and SP600125 (SP) respectively, on the caspase-3 activation and cell death induced by AA in Caco- 2 cells. Thus, cells were pre-treated during $1 \mathrm{~h}$ with $1 \mathrm{mM}$ of NAC or $50 \mu \mathrm{M}$ PD or $50 \mu \mathrm{M}$ SP before the AA treatment during $8 \mathrm{~h}$ for caspase- 3 assays and $16 \mathrm{~h}$ for cell viability determination. As shown in Fig. 4A, the AAinduced caspase- 3 activity was significantly decreased by pre-treatment of Caco- 2 cells with SP and NAC but not with PD. Similarly, the inhibition of JNKs or the presence of NAC, but not the inhibition of ERKs, resulted in the suppression of AA-induced Caco-2 cell death (Fig. 4B). Additionally, treatment with NAC or inhibitors alone for $24 \mathrm{~h}$ had no significant effects on the viability of cells compared to the untreated group (data not shown). Consequently, these results indicate that both oxidative stress and activation of JNKs seem to play a role in the signal transduction leading to apoptosis and cell death induced by AA in Caco-2 cells.

3.5. Effect of EC, PB2 and CPE on apoptosis and cytotoxicity induced by $A A$. 
Since dietary flavonoids have shown to function as powerful antioxidants, we next examined the ability of a well-standardized cocoa polyphenolic extract (CPE) and its main polyphenol components $\mathrm{EC}$ and $\mathrm{PB} 2$ to protect against AA-induced apoptosis and cytotoxicity. The working concentrations of the polyphenols were fixed at $10 \mu \mathrm{g} / \mathrm{mL}$ for the $\mathrm{CPE}$ and $10 \mu \mathrm{M}$ for $\mathrm{EC}$ and $\mathrm{PB} 2$, which represent realistic concentrations in the human gut. To assess whether treatment with dietary polyphenols repressed the cytotoxic effect induced by AA, Caco-2 cells were treated for $20 \mathrm{~h}$ with EC, PB2 or CPE and, after removing polyphenol-containing culture medium, cells were further exposed to $5 \mathrm{mM} \mathrm{AA}$; then, activation of caspase- 3 and cell death were evaluated at $8 \mathrm{~h}$ and $16 \mathrm{~h}$, respectively. Fig. $5 \mathrm{~A}$ shows that treatment of cells with EC, PB2 or CPE significantly decreased the AAincreased caspase- 3 activity reaching control values in the case of PB2 and CPE. Similarly, EC, PB2 and CPE significantly protected against AA-induced cell death induced by AA (Fig. 5B) although this protection was lower with EC. Altogether, these results seem to indicate that treatment of Caco-2 cells with $\mathrm{PB} 2$ or $\mathrm{CPE}$ totally protects against AAinduced apoptosis and cell death while treatment with EC exerted a slight protective action.

\subsection{Effect of EC, PB2 and CPE treatment on GSH depletion and ROS formation induced} by $A A$.

To better understand the mechanisms by which cocoa polyphenols protects against AA-induced apoptosis and cytotoxicity, we next evaluated the effect of the pre-treatment of Caco-2 cells with EC, PB2 and CPE on the redox status induced by AA. Caco-2 cells were pre-treated during 20 hours with $10 \mu \mathrm{M}$ of EC or PB2 or with $10 \mu \mathrm{g} / \mathrm{mL}$ of CPE before the $5 \mathrm{mM}$ AA treatment. As shown in Figure 6A, pre-treatment of Caco-2 cells with cocoa polyphenols significantly prevented the depletion of GSH induced by 2 hours of $5 \mathrm{mM}$ AA. 
Similarly, ROS production at 4 hours was significantly decreased in cells pre-treated with EC, PB2 or CPE as compared to AA-treated cells (Figure 6B). In fact, PB2- and CPEtreated cells showed levels similar to those of control cells. These results indicate that treatment with cocoa polyphenols prevented GSH depletion and ROS generation induced by AA. Interestingly, once again, this prevention was higher with PB2 and CPE than with EC.

\subsection{PB2 and CPE treatment increases the GST and $\gamma$-GCS protein levels.}

The induction of antioxidant/detoxification enzymes has been suggested to be a further mechanism of polyphenols to improve the intracellular defences and protect against oxidative stress insults. Therefore, we next evaluated the effect of AA treatment in control and EC-, PB2- and CPE-treated cells on the rate-limiting enzyme in GSH synthesis, $\gamma$-GCS and the detoxification enzyme GST. Likewise, we also determined the effect of EC, PB2 and CPE pre-treatment on the levels of both enzymes. To this end, Caco-2 cells were pretreated during $20 \mathrm{~h}$ with $10 \mu \mathrm{M}$ of EC or PB2 or with $10 \mu \mathrm{g} / \mathrm{mL}$ of CPE. After that, control and polyphenol-treated cells were exposed to $5 \mathrm{mM} \mathrm{AA}$ and the levels of $\gamma$-GCS and GST were evaluated by Western blotting at different times (0-16 h). As shown in Fig. 7, the protein levels of both enzymes significantly decreased after $4 \mathrm{~h}$ of AA treatment and continuously decreased until $16 \mathrm{~h}$ in control cells. EC treatment did not affect $\gamma$-GCS and GST basal levels but avoided the decrease on $\gamma$-GCS levels and delayed until 8 hours the decrease on GST levels induced by AA. On the other hand, $\gamma$-GCS and GST levels were increased in PB2- and CPE-treated cells before the AA treatment as compared with control cells and, interestingly, they remained increased in the presence of AA. These results 
indicate that treatment of Caco-2 cells with PB2 and CPE increased the cellular levels of $\gamma$ GCS and GST and avoided the decrease in both enzymes induced by AA.

\subsection{PB2 and CPE treatment prevent the phorphorylation of JNKs induced by AA.}

Finally, to verify that the improved redox status induced by PB2 and CPE in Caco-2 cells was correlated with the prevention of apoptotic signalling induced by AA, we investigated the effect of the treatment with cocoa polyphenols in the signalling pathways activated by AA. To this end, Caco- 2 cells treated during 20 hours with EC, PB2 and CPE were further exposed to $5 \mathrm{mM}$ of AA for different times $(0-16 \mathrm{~h})$ and then immunoblots were performed using phospho- and non-phospho-antibodies against ERKs and JNKs. Fig. 8A shows that before AA treatment the p-ERK levels were increased in EC-treated cells although they returned to control levels after $8 \mathrm{~h}$ of AA treatment. In addition, similar to what was found in control cells treated with AA (Fig. 3), the EC-treated cells showed an increase in p-JNKs levels after $4 \mathrm{~h}$ of AA treatment and remained active until $16 \mathrm{~h}$. On the other hand, PB2- and CPE-treated cells also showed an increase in p-ERKs levels before the AA treatment but in this case, the ERK activation continued in the presence of AA for the length of the experiment. In addition, contrary to control and EC-treated cells, there were no changes in p-JNKs levels in PB2- and CPE-treated cells in the presence of AA (Fig. 8B and C). Likewise, there was no difference in the total levels of ERKs or JNKs in any treatment.

Altogether, these results indicate that EC, PB2 and CPE treatment activate the ERK pathway and continued increased in the presence of AA only in PB2- and CPE-treated cells. Similarly, PB2 and CPE treatment also prevented the increase of p-JNKs levels induced by AA. 


\section{Discussion}

It has been recently shown in humans that regular consumption of products containing AA increases the production of reactive oxygen radicals in leukocytes and induces a generalized pro-inflammatory state [4]. These observations, together with the evidences of AA-induced apoptosis both in animals [24,25] and in cell cultures [26,27], suggest a relationship between oxidative stress and cell damage in the presence of this toxic. To our knowledge, the present study is the first to show that AA-induced oxidative stress leads to apoptosis and cytotoxicity in human intestinal Caco-2 cells. This is important since ingested AA is rapidly absorbed to a high degree in the human intestine [11] and, therefore, studies assessing AA influence on gastrointestinal cells integrity seem necessary. Interestingly, we also demonstrate that physiological concentrations of dietary antioxidant compounds like procyanidin B2 and a mixture of cocoa polyphenols were able to counteract the deleterious effect of AA. Thus, an increased intake of these natural antioxidants could be relevant to mitigate AA-associated risks related to oxidative stress.

GSH is the most abundant cellular antioxidant responsible for the maintenance of the cellular redox state. Consequently, GSH depletion induced by AA may contribute to the creation of oxidative stress conditions as previously demonstrated in HepG2 cells [7] and in human-derived monocytes-macrophages in vitro [4]. Our results show that treatment of Caco-2 cells with AA led to a significant depletion of GSH and overproduction of ROS, and consequently to an increase in oxidative stress. In addition, we found that redox disturbance precedes activation of caspase-3, considered a very specific and sensitive apoptotic marker [28], suggesting a temporal link between GSH depletion, ROS overproduction and apoptotic cell death induced by AA in Caco- 2 cells. This association was confirmed by the fact that pre-treatment with NAC, a potent GSH precursor, abolishes 
apoptosis and cytotoxicity generated by AA. Therefore, as previously shown in Caco-2 cells $[13,14]$ an in other cell types $[29,30]$, the loss of cellular GSH redox status seems to be an important player in apoptotic signalling.

AA-induced apoptosis has already been demonstrated in the rat nervous system [24] as well as in neuroblastoma [26] and astrocytoma [27] cells. However, the modes of action underlying this effect have not been clearly defined. One of the potential mechanisms involved could be the activation of signalling pathways implicated in the apoptotic process. In this regard, numerous investigations have pointed out the importance of the MAPK pathway in the regulation of stress-dependent apoptosis [10]. In addition, ROS overproduction activates JNK- and ERK-MAPKs to induce apoptotic reactions in oxidative stress-induced intestinal epithelial cell injury [31]. Interestingly, we found that following ROS generation, AA caused a rapid but transient activation of ERKs and a persistent activation of JNKs in Caco-2 cells. In general, activation of ERKs seems to favour survival signals, while sustained JNKs activation results in apoptosis [10]. Supporting this, in this work specific JNKs but not ERKs inhibitors prevented the subsequent increase in caspase-3 activity and cell death induced by AA, indicating that JNKs activation participated in the mechanism of AA induced apoptosis. Further support for this hypothesis is provided by previous findings of induction of JNKs contributing to the differentiation and apoptotic pathways in Caco-2 cells [32]. Moreover, enhanced expression and activation of JNKs has also been related with intestinal inflammatory conditions [33,34] thus, these results indicate that AA ingestion may constitute a dietary risk factor for intestine injury.

Several natural products have been shown to protect cells against oxidative damage by virtue of their antioxidant properties [35]. Among them, cocoa flavonoids and their metabolites have demonstrated to possess antioxidant capacity and to protect against an 
oxidative insult [36]. Indeed, polyphenols and especially PB2 are poorly absorbed in the intestine so its beneficial effects would be restricted to the gastrointestinal tract where they may have an important local function neutralising oxidants [36]. For example, cocoa procyanidins have been recently reported to protect intestinal Caco- 2 cell from the loss of integrity induced by a lipophilic oxidant [37]. In addition, high cocoa intake has also been shown to modulate intestinal and systemic immune cell functionality in vivo [38]. Accordingly, the present study shows that a complex mixture of cocoa polyphenols and the pure flavanols PB2 and EC effectively suppressed the increase in caspase- 3 activity and the cell death induced by AA in caco-2 cells.

An interesting observation in the present study is the finding that the protective effect of CPE and PB2 against AA cytotoxicity was higher than that of EC. Furthermore, both CPE and PB2 almost completely blocked the decrease of GSH induced by AA and totally abrogated the subsequently increased ROS generation while these effects were only partially restored with EC. This result suggests that the minor effect exerted by EC could be partially ascribed to the fact that EC mainly acted as scavenger of free radicals. However, similar to what was reported for other polyphenols and antioxidants [39], PB2 and CPE could protect cell constituents not only by neutralising several types of radicals but also by up-regulating antioxidant defences as well as interacting with signalling pathways involved in cell survival.

To further corroborate this latter hypothesis, we evaluated the effect of cocoa polyphenols and AA in the protein levels of $\gamma$-GCS and GST enzymes. $\gamma$-GCS catalyses the first and rate limiting step in GSH synthesis while GST is involved in the detoxification of xenobiotics. Both GST and GSH inducers could be potential inhibitors of AA cytotoxicity. According to that, we found that PB2 and CPE but not EC increased the basal 
levels of $\gamma$-GCS and GST, and significantly prevented the AA-induced decrease in both enzymes. Therefore, these results support the important role of the induction of cytoprotective enzymes exhibited by some polyphenols as an additional mechanism of action to better defend against AA insult. Similar results have been recently shown with phenolic compounds increasing glutathione related enzymes in intestine to protect against oxidative stress induced by lipid peroxidation [40].

Consistent with the above observations, AA-induced activation of JNKs was also blocked by PB2 and CPE treatment but not by EC. Interestingly, there is a relationship between elevated GST expression and resistance to apoptosis indicating the critical role that GST plays in modulating JNK signalling pathways [41]. In this line, our results agree with other studies in which increased GST expression results in decreased JNK activity and protection from ROS-mediated cell death $[41,42]$. Furthermore, the increased activation of ERKs shown with all polyphenol probably plays an indirect protective role by these compounds through the promotion of cell proliferation and survival signalling. Therefore, we suggest that the modulation of GSH levels through the fortifying of cellular defences along with the induction of survival pathways by PB2 and CPE inhibit the AA-induced intracellular signals leading to apoptosis and results in cell survival. All together, these results confirm the antiapoptotic effect of these dietary compounds as a further mechanism of action.

It is worth noting that the concentrations of AA applied to the cell cultures in the present study were to some extent high; however, other authors [4] and initial studies in our laboratory have showed that smaller doses of AA can act as toxicant because similar effects could be observed under long-term exposure. For methodological reasons, the actual conditions of cell culture favoured to increase the dose and reduce the exposure time and 
this has to be taken into consideration when further discussing the consequences for the dietary intake of AA-containing food. On the other hand, we can not rule out that daily exposure to a lower dose of AA may bring about a risk factor of toxicity in human. Therefore, efforts designed both to reduce the AA content of processed foods and to mitigate adverse manifestation of AA after consumption are necessary.

In summary, our present study provides evidence that redox perturbation induced by AA leads to activation of JNKs, increased caspase-3 activity and enhanced apoptosis and cytotoxicity in Caco-2 cells. Interestingly, AA cytotoxicity was fully counteracted by dietary phenols such as CPE or PB2 which prevented GSH depletion and ROS overproduction, and blocked the apoptotic pathways activated by AA. Furthermore, both compounds exhibited antioxidant properties by modulating the levels of cytoprotective enzymes such as $\gamma$-GCS and GST and anti-apoptotic effects by influencing signalling pathways implicated in apoptosis and survival. All together, these results strongly support the role of the redox status in AA-mediated apoptosis and the potential contribution of this process to the genesis of gut pathology. In addition, they underline the important role of dietary antioxidants like cocoa polyphenols for nutritional prevention of certain pathological and physiological states linked to oxidative stress. 


\section{References}

[1] Tareke E, Rydberg P, Karlsson P, Eriksson M, Tornqvist M. Analysis of acrylamide, a carcinogen formed in heated food-stuffs. J Agric Food Chem. 2002; 50:4998-5006.

[2] Parzefal W. Minireview on the toxicity of dietary acrylamide. Food Chem Toxicol. 2008; 46:1360-1364

[3] Park H, Kim M-S, Kim SJ, Park M, Kong KH, Kim HS, Kwack SJ, Kang TS, Kim SH, Kim HS, Lee J. Acrylamide induces cell death in neural progenitor cells and impairs hippocampal neurogenesis. Toxicol Lett. 2010; 193:86-93

[4] Naruszewicz M, Zapolska-Downar D, Kosmider A, Nowicka G, KozłowskaWojciechowska M, Vikstrom AS, Tornqvist M. Chronic intake of potato chips in humans increases the production of reactive oxygen radicals by leukocytes and increases plasma $\mathrm{C}$ reactive protein: a pilot study. Am J Clin Nutr 2009; 89:773-7.

[5] Zhang X, Cao J, Jiang L, Geng Ch, Zhong L. Protective effect of hydroxytyrosol against acrylamide-induced cytotoxicity and DNA damage in HepG2 cells. Mutat Res. $2009 ; 664: 64-68$.

[6] Yousef MI, El-Demerdash FM. Acrylamide-induced oxidative stress and biochemical perturbations in rats. Toxicology 2006; 219:133-141

[7] Cao J, Liu Y, Jia L, Jiang L-P, Geng Ch, Yao X-F, Kong Y, Jiang B-N, Zhong L. Curcumin attenuates acrylamide-induced cytotoxicity and genotoxicity in HepG2 cells by ROS Scavenging. J Agric Food Chem 2008; 56:12059-63

[8] Pernice R, Hauder J, Koehler P, Vitaglione P, FoglianoV, Somoza V. Effect of sulforaphane on glutathione-adduct formation and on glutathione_S_transferase-dependent detoxification of acrylamide in Caco-2 cells. Molec Nutr Food Res. 2009; 53:1540-1550 
[9] Zhu YJ, Zeng T, Zhu YB, Yu SF, Wang QS, Zhang LP, Guo X, Xie KQ. Effects of acrylamide on the nervous tissue antioxidant system and sciatic nerve electrophysiology in the rat. Neurochem Res. 2008; 11:2310-2317.

[10] Valko M, Leibfritz D, Moncol J, Cronin MT, Mazur M, Telser J. Free radicals and antioxidants in normal physiological functions and human disease. Int J Biochem Cell Biol. $2007 ; 39: 44-84$

[11] Zödl B, Schmid D, Wassler G, Gundacker C, Leibetseder V, Thalhammer T, Ekmekcioglu C. Intestinal transport and metabolism of acrylamide. Toxicology 2007; 232:99-108.

[12] Lash LH. Mitochondrial glutathione transport: physiological, pathological and toxicological implications. Chem Biol Interact. 2006; 163:54-67

[13] Circu ML, Rodriguez C, Maloney R, Moyer MP, Aw TY. Contribution of mitochondrial GSH transport to matrix GSH status and colonic epithelial cell apoptosis. Free Radical Bio Med. 2008; 44: 768-778.

[14] Wang TG, Gotoh Y, Jennings MH, Rhoads CA, Aw TY. Lipid hydroperoxide-induced apoptosis in human colonic CaCo-2 cells is associated with an early loss of cellular redox balance. FASEB J. 2000; 14:1567-1576

[15] Lamuela-Raventós RM, Romero-Pérez AI, Andrés-Lacueva C, Tornero A. Health Effects of Cocoa Flavonoids. Food Sci Technol Int. 2005; 11:159-176

[16] Vinson JA, Proch J, Bose P, Muchler S, Taffera P, Shutta D, Samman N, Agbor GA. Chocolate is a powerful ex vivo and in vitro antioxidant, antiatherosclerotic agent in an animal model, and a significant contributor to antioxidants in the European and American diets. J Agric Food Chem. 2006; 54:8071-8076. 
[17] Martín MA, Ramos S, Mateos R, Granado-Serrano AB, Izquierdo-Pulido M, Bravo L,

Goya L. Protection of human HepG2 cells against oxidative stress by cocoa phenolic extract. J Agric Food Chem. 2008; 56:7765-72.

[18] Martín MA, Granado-Serrano AB, Ramos S, Izquierdo-Pulido M, Bravo L, Goya L. Cocoa flavonoids up-regulate antioxidant enzyme activity via the ERK1/2 pathway to protect against oxidative stress-induced apoptosis in HepG2 cells. J Nutr Biochem. 2010; 21:196-205

[19] Granado-Serrano AB, Martín MA, Goya L, Bravo L, Ramos S. Time-course regulation of survival pathways by epicatechin on HepG2 cells. J Nutr Biochem. 2009; 20:115-124 [20] Granado-Serrano AB, Martín MA, Izquierdo-Pulido M, Goya L, Bravo L, Ramos S. Molecular mechanisms of (-)-epicatechin and chlorogenic acid on the regulation of the apoptotic and survival/proliferation pathways in a human hepatoma cell line. J Agric Food Chem. 2007; 55:2020-2027

[21] Alía M, Ramos S, Mateos R, Granado-Serrano AB, Bravo L, Goya L. Quercetin protects human hepatoma cell line (HepG2) against oxidative stress induced by tertbutyl hydroperoxide. Toxicol Appl Pharmacol 2006; 212:110-118

[22] Granado-Serrano AB, Martin MA, Bravo L, Goya L, Ramos S. Quercetin induces apoptosis via caspase activation, regulation of $\mathrm{Bcl}-2$, and inhibition of PI-3-kinase/Akt and ERK pathways in a human hepatoma cell line (HepG2). J Nutr. 2006; 136:2715-2721.

[23] Hissin PJ, Hilf R. A fluorometric method for determination of oxidised and reduced glutathione in tissues. Anal Biochem 1976; 74:214-226.

[24] Li S, Cui N, Zhang C, Zhao S, Yu S, Xie K. Effect of subchronic exposure to acrylamide induced on the expression of bcl-2, bax and caspase- 3 in the rat nervous system. Toxicology 2006; 217: 46-53 
[25] Zhang X, Zhao C, Jie B. Various dietary polyunsaturated fatty acids modulate acrylamide-induced preneoplasic urothelial proliferation and apoptosis in mice. Experimental Toxicol Pathol. 2010; 62:9-16

[26] Sumizawa T, Igisu H. Apoptosis induced by acrylamide in SH-SY5Y cells. Arch Toxicology 2007; 81:279-282

[27] Chen JH, Wub K-Y, Chiu I-M, Tsou T-C, Chou Ch-Ch. Acrylamide-induced astrogliotic and apoptotic responses in human astrocytoma cells. Toxicol in Vitro 2009; 23:855-861.

[28] Gomez-Lechon MJ, O’Connor E, Castell JV, Jover R. Sensitive markers used to identify compounds that trigger apoptosis in cultured hepatocytes. Toxicol Sci. 2002; 65: 299-308.

[29] Esteve JM, Mompo J, de la Asuncion JA, Sastra J, Asensi M, Boix J, Vina JR, Pallardo FV. Oxidative damage to mitochondrial DNA and glutathione oxidation in apoptosis: studies in vivo and in vitro. FASEB J. 1999; 13:1055-1064

[30] Ekshyyan O, Aw TY. Decreased susceptibility of differentiated P12 cells to oxidative challenge: relationship to cellular redox and expression of apoptotic protease activator factor-1. Cell Death Differ. 2005; 12:1066-1077.

[31] Zhou Y, Wang O, Evers BM, Chung DH. Signal transduction pathways involved in oxidative stress-induced intestinal epithelial cell apoptosis. Pediatr Res. 2005; 58:11921197.

[32] Ding Q, Wang Q, Evers BM. Alterations of MAPK activities associated with intestinal cell differentiation. Biochem Bioph Res Commun. 2001; 284:282-288 
[33] Zingarelli B, Yang Z, Hake PW, Denenberg A, Wong HR. Absence of endogenous interleukin-10 enhances early stress response during postischemic injury in mice intestine. Gut 2001; 5: 610-22.

[34] Romier B, Schneider Y-J, Larondelle Y, During A. Dietary polyphenols can modulate the intestinal inflammatory response. Nutr Rev. 2009; 67: 363-378

[35] Virgili F, Marino M. Regulation of cellular signals from nutritional molecules: a specific role for phytochemicals, beyond antioxidant activity. Free Radical Biol Med. 2008; $45: 1205-1216$

[36] Ramiro-Puig E, Castell M. Cocoa: antioxidant and immunomodulator. Brit J Nutr. 2009; 101: 931-940

[37] Erlejman AG, Fraga CG, Oteiza PI. Procyanidins protect Caco-2 cells from Bile acidand oxidant-induced damage. Free Radic Biol Med. 2006; 41: 1247-1256.

[38] Ramiro-Puig E, Perez-Cano FJ, Ramos-Romero S, Pérez-Berezo T, Castellote C, Permanyer J, Franch A, Izquierdo-Pulido M, Castell M. Intestinal immune system of young rats influenced by cocoa enriched diet. J Nutr Biochem 2008; 19: 555-565.

[39] Masella RT, Di Benedetto R, Varı R, Filesi C, Giovannini C. Novel mechanisms of natural antioxidant compounds in biological systems: involvement of glutathione and glutathione-related enzymes. J Nutr Biochem. 2005; 16: 577-586.

[40] Giovanninia C, Scazzocchio B, Matarrese P, Varıa R, D’Archivio M, Di Benedetto R, Casciani S, Dessi MR, Straface E, Malorni W, Masella R. Apoptosis induced by oxidized lipids is associated with up-regulation of p66Shc in intestinal Caco-2 cells: protective effects of phenolic compounds. J Nutr Biochem. 2008; 19: 18- 128 
[41] Romero L, Andrews K, Ng L, O’rourke K, Maslen A, Kirby G. Human GSTA1-1 reduces c-Jun N-terminal kinase signalling and apoptosis in Caco-2 cells. Biochem J. 2006; 400: $135-141$

[42] Yin Z, Ivanov VN, Habelhah H, Tew K, Ronai Z. Glutathione S-transferase elicits protection against $\mathrm{H} 2 \mathrm{O} 2$-induced cell death via coordinated regulation of stress kinases. Cancer Res. 2000; 60: 4053-4057 


\section{Legends to Figures}

Figure 1.- Effect of AA on cell viability and caspase-3 activity. Caco-2 cells were treated during 16 hours with the noted concentrations of AA and cell viability was determined as relative percentage of viable cells (A) and as LDH leakage (B). Caco-2 cells were incubated during 8 hours with increasing concentrations of 1-20 mM AA and caspase-3 activity was assayed (C). Data represent means \pm SD of 10-12 samples per condition. Different letters denote statistically significant differences, $P<0.05$.

Figure 2.- Effect of AA on GSH levels and ROS generation. Caco-2 cells were treated with the indicated concentrations of AA and GSH levels were determined at 1,2 and 4 hours of incubation (A). Caco-2 cells were treated with the noted concentrations of AA and intracellular ROS production was evaluated at 0, 1, 2, 3 and 4 hours (B). Values are means \pm SD of 10-12 different samples per condition. Different letters denote statistically significant differences, $P<0.05$.

Figure 3.- Effect of AA on total and phosphorylated levels of ERKs and JNKs. Caco-2 cells exposed to $5 \mathrm{mM}$ AA for the indicated times were subjected to Western blot analysis using total and phospho-specific antibodies to ERKs or JNKs. Representative Western blot (A) and percentage values (B) of p-ERKs/ERKs and p-JNKs/JNKs relative to the control conditions of three different experiments (means \pm SD). Means without a common letter differ, $P<0.05$. 
Figure 4.- Effect of N-acetilcysteine (NCA) and selective inhibitors PD98059 (PD) and SP600125 (SP) on caspase-3 activation and cell death induced by AA. Caco-2 cells were pre-treated with $1 \mathrm{mM}$ NAC or $50 \mu \mathrm{M}$ PD or $50 \mu \mathrm{M}$ SP. Then, cells were further exposed to $5 \mathrm{mM} \mathrm{AA}$ in the presence of NAC, PD and SP, and caspase- 3 activity (A) and cell viability (B) were determined at $8 \mathrm{~h}$ and $16 \mathrm{~h}$, respectively. Data represent means $\pm \mathrm{SD}$ of 10 separate experiments. Different letters denote statistically significant differences, $P<$ 0.05 .

Figure 5.- Protective effect of EC, PB2 and CPE on caspase-3 activation and cell death induced by AA. Caco- 2 cells were incubated with $10 \mu \mathrm{M}$ of EC and PB2 and $10 \mu \mathrm{g} / \mathrm{mL}$ of CPE for 20 hours. Control and polyphenol-treated cells were further exposed to $5 \mathrm{mM} \mathrm{AA}$, after removing polyphenol-containing culture medium, and caspase-3 activity (A) and cell viability (B) were determined at 8 and 16 h, respectively. Data represent means \pm SD of 10 separate experiments. Different letters denote statistically significant differences, $P<0.05$.

Figure 6.- Effect of EC, PB2 and CPE on the decreased GSH levels and increased ROS generation induced by AA. Caco- 2 cells were treated with $10 \mu \mathrm{M}$ of EC and PB2 and 10 $\mu \mathrm{g} / \mathrm{mL}$ of CPE for 20 hours and further exposed to $5 \mathrm{mM} \mathrm{AA}$. GSH levels (A) and ROS generation (B) were determined in control and polyphenol-treated cells after 2 and $4 \mathrm{~h}$ of AA treatment, respectively. Data represent means \pm SD of 10-12 samples per condition. Different letters denote statistically significant differences, $P<0.05$

Figure 7.- Effect of EC, PB2 and CPE on GST and $\gamma$-GCS protein levels. Caco-2 cells were incubated with $10 \mu \mathrm{M}$ of EC and PB2 and $10 \mu \mathrm{g} / \mathrm{mL}$ of CPE for 20 hours. Control 
and EC- PB2- and CPE-treated cells were further exposed to $5 \mathrm{mM}$ AA for the indicated times and subjected to Western blot analysis using specific antibodies to $\gamma$-GCS and GST. Representative Western blot (A) and percentage values of $\gamma$-GCS and GST (B) of three different experiments relative to the control conditions at time 0 (means $\pm \mathrm{SD}$ ). Normalization of Western blots was ensured by $\beta$-actin. Means without a common letter differ, $P<0.05$

Figure 8.- Effect of EC, PB2 and CPE on total and phosphorylated levels of ERKs and JNKs. Caco-2 cells were incubated with $10 \mu \mathrm{M}$ of EC and PB2 and $10 \mu \mathrm{g} / \mathrm{mL}$ of CPE for 20 hours. Control and polyphenol-treated cells were further exposed to $5 \mathrm{mM}$ AA for the indicated times and subjected to Western blot analysis using total and phospho specific antibodies to ERKs or JNKs. Representative Western blot and percentage values of pERKs/ERKs and p-JNKs/JNKs of three different experiments relative to the control conditions (means \pm SD) in EC- (A), PB2- (B) and CPE-treated cells (C). Normalization of Western blots was ensured by $ß$-actin. Means without a common letter differ, $P<0.05$ 